University of Nebraska - Lincoln

DigitalCommons@University of Nebraska - Lincoln

\title{
Estimating Mandibular Motion Based on Chin Surface Targets During Speech
}

Jordan R. Green

University of Nebraska-Lincoln, jgreen4@unl.edu

Erin M. Wilson

Waisman Center

Yu-Tsai Wang

National Yang-Ming University

Christopher A. Moore

University of Washington - Seattle Campus

Follow this and additional works at: https://digitalcommons.unl.edu/specedfacpub

Part of the Special Education and Teaching Commons

Green, Jordan R.; Wilson, Erin M.; Wang, Yu-Tsai; and Moore, Christopher A., "Estimating Mandibular Motion Based on Chin Surface Targets During Speech" (2007). Special Education and Communication Disorders Faculty Publications. 7.

https://digitalcommons.unl.edu/specedfacpub/7

This Article is brought to you for free and open access by the Department of Special Education and Communication Disorders at DigitalCommons@University of Nebraska - Lincoln. It has been accepted for inclusion in Special Education and Communication Disorders Faculty Publications by an authorized administrator of DigitalCommons@University of Nebraska - Lincoln. 
Published in Journal of Speech, Language, and Hearing Research 50 (August 2007), pp. 928-939; doi: 10.1044/1092-4388(2007/066)

Copyright (c) 2007 American Speech-Language-Hearing Association. Used by permission.

Submitted June 2, 2006; accepted January 2, 2007.

Research Note

\title{
Estimating Mandibular Motion Based on Chin Surface Targets During Speech
}

\author{
Jordan R. Green, University of Nebraska-Lincoln \\ Erin M. Wilson, Waisman Center \\ Yu-Tsai Wang, School of Dentistry, National Yang-Ming University, Taipei, Taiwan \\ Christopher A. Moore, University of Washington, Seattle \\ Corresponding author - Jordan R. Green, Department of Special Education and Communication Disorders, \\ University of Nebraska-Lincoln, 318 Barkley Center, Lincoln, NE 68583; email jgreen4@unl.edu
}

\begin{abstract}
Purpose: The movement of the jaw during speech and chewing has frequently been studied by tracking surface landmarks on the chin. However, the extent to which chin motions accurately represent those of the underlying mandible remains in question. In this investigation, the movements of a pellet attached to the incisor of the mandible were compared with those of pellets attached to different regions of the chin.

Method: Ten healthy talkers served as participants. Three speaking contexts were recorded from each participant: word, sentence, and paragraph. Chin position errors were estimated by computing the standard distance between the mandibular incisor pellet and the chin pellets.

Results: Relative to the underlying mandible, chin pellets moved with an average absolute and relative error of $0.81 \mathrm{~mm}$ and $7.30 \%$, respectively. The movements of chin and mandibular pellets were tightly coupled in time.

Conclusion: The chin tracking errors observed in this investigation are considered acceptable for descriptive studies of oromotor behavior, particularly in situations where mandibular placements are not practical (e.g., young children or edentulous adults). The observed amount of error, however, may not be tolerable for finegrained analyses of mandibular biomechanics. Several guidelines are provided for minimizing error associated with tracking surface landmarks on the chin.
\end{abstract}

Keywords: jaw motion, speech motor control, skin motion artifact, mandible

Empirical studies of jaw movement in humans are of interest to a large number of disciplines including dentistry, occupational therapy, orthodontics, psychology, and speech language-pathology. Jaw movements ${ }^{1}$ during speech and chewing have been recorded using a number of technologies such as x-ray (e.g., Ahlgren, 1966; Kuehn, Reich, \& Jordan, 1980); x-ray microbeam (e.g., Abbs, Nadler, \& Fujimura, 1988; Westbury, 1991); strain gauge (e.g., Abbs \& Gilbert, 1973; Sussman, MacNeilage, \& Hanson, 1973); magnetometry (Hixon, 1971; Perkell, Cohen, Svirsky, Matthies, Garabieta, \& Jackson, 1992); and video and optical tracking (Green, Moore, Hi- gashikawa, \& Steeve, 2000; A. Smith, Goffman, Zelaznik, Ying, \& McGillem, 1995). Obtaining information about jaw movement during speech and chewing has, however, been particularly challenged by the mandible's inaccessibility. Consequently, jaw movements during these behaviors have been frequently described on the basis of the movements of chin surface landmarks (e.g., Barlow, Cole, \& Abbs, 1983; Caruso, Stanhope, \& McGuire, 1989; DeNil, \& Abbs, 1991; Gracco, 1988, 1994; Hixon, 1971; Kelso, Vatikiotis-Bateson, Saltzman, \& Kay, 1985; Häggman-Henrikson, Eriksson, Nordh, \& Zafar, 1998; Hamlet, \& Stone, 1978; McFarland \& Lund, 1995; Müller \&

\footnotetext{
${ }^{1}$ Throughout the manuscript, mandible refers to the mandibular bone, chin refers to the skin overlying the bony mandible, and jaw is used when a distinction between chin and mandible is not required.
} 
Abbs, 1979; Shaiman, 2002; Sharkey \& Folkins, 1985; B. L. Smith \& McLean-Muse, 1987; Stone, 1981; Sussman \& Smith, 1970; Throckmorton, Buschang, Hayasaki, \& Phelan, 2001).

Recent developments in computer pattern recognition have made high-speed optical motion capture systems an attractive option for registering jaw movements. Participants in these studies are not exposed to radiation, are not required to maintain restrictive postures, and are not encumbered by wires or cantilever beams extending from the articulators. Moreover, most participants are unaware of the small $(\sim 2 \mathrm{~mm})$ markers that are used to track facial motion. Currently, video and optical motion capture systems provide the only suitable method for studying jaw movements in children under the age of 4 years (e.g., Green \& Wilson, 2006).

Unfortunately, the reliability and validity of using chin movements to represent those of the underlying mandible are not fully known. Using magnetometry to transduce jaw movements, Hixon (1971) reported that the movement patterns of the chin were highly consistent across multiple repetitions of /apa/ produced by a single participant. Prior findings, which tracked jaw movements using video motion capture, suggested that chin movements are highly reliable (Green, Moore, \& Reilly, 2002). In this prior study, vertical chin movements from 10 adult participants were recorded as they produced 10 consecutive productions of a basic speech utterance. The 10 movement traces obtained from each participant were highly correlated $(r=.97)$, which indicated that the participants' movement patterns were consistent across trials and that the movement tracking system was highly reliable.

Although reliability appears to be acceptable for this method, the extent to which chin motions accurately represent mandibular motions during speech and chewing has been questioned (Cooker, 1973; Jemt \& Hedgård, 1982; Häggman-Henrikson et al., 1998; Kuehn, Reich, \& Jordan, 1980). Skin-motion artifacts are a problem inherent to all studies that rely on the tracking of superficially mounted skin markers for the study of skeletal motion (Holden, Stanhope, \& Orsini, 1994). Unlike the mandible, the motions of the chin are affected by the combined influences of inertia, the biomechanic and viscoelastic properties of facial tissues, and movements of the lower lip. These factors have the potential to influence both the spatial and temporal aspects of chin movement.

Several investigators have estimated the positional differences between chin and mandibular movements during speech. Using cineradiography, Kuehn and colleagues (1980) measured positional differences between the mandible and several chin markers in 3 participants during a variety of speaking tasks. Their results indicated that across participants, the average standard deviation between the vertical positions (relative to the head) of chin and mandible markers ranged from $1.03 \mathrm{~mm}$ to
$1.65 \mathrm{~mm}$, and the average standard deviation for the anterior-posterior dimension across participants ranged from $0.55 \mathrm{~mm}$ to $1.19 \mathrm{~mm}$. Using strain gauge technology, Cooker (1973) observed that the chin moved significantly in response to movements of the lower lip in 4 male talkers. Differences between the displacements of the chin and the mandible ranged between $0.4 \mathrm{~mm}$ and $2.1 \mathrm{~mm}$, depending on vowel context. Cooker also reported that the extent to which motions of the lower lip affect chin skin varies for different locations, with skin overlying the angle of the mandible being less affected than skin near the chin's midline.

A more current estimate of the difference between chin and mandible motions during speech is warranted in light of the significant instrumental advances since the reports of Cooker (1973) and Kuehn and colleagues (1980), which relied on strain gauge transduction and hand tracings of the mandible on cineradiographic films, respectively. This investigation reports positioning and timing differences between multiple pellets mounted to the chin and mandible using $\mathrm{x}$-ray microbeam (XRMB) technology (see Westbury, 1994). The ease of data collection and postprocessing using the XRMB facilitated the study of more participants, speaking tasks, and chin regions than have been studied in the past.

This report also examines the strengths and weaknesses of different representations of chin movement in time. Because the mandible rotates and translates during speech (Edwards \& Harris, 1990; Ostry \& Munhall, 1994; Vatikiotis-Bateson \& Ostry, 1995), there is no error-free method for representing jaw motion in time using a single dimension. Specifically, projection distortions are contained in both unidimensional (e.g., vertical or anterior-posterior) and derived representations of jaw movement, which are obtained typically using a principle component analysis or the Euclidean distance transform. For example, transforming 2D data into a single variable that represents a pellet's Euclidean distance from the origin will erroneously register no movement when a pellet rotates with a constant radius about the origin. Therefore, to identify the signal that contains the best representation of mandibular movement (i.e., smallest amount of skin movement error), comparisons between chin and mandibular movements were made for three different signals: anterior-posterior movement $(X)$, vertical movement $(\mathrm{Y})$, and the Euclidean distances (D) from the origin of a head-based anatomical coordinate system.

\section{Method}

\section{Participants}

Ten healthy talkers served as participants ( 5 men, 5 women) with a mean age of 20/3 [years/months] (range 
= 19-34 years). Participants reported negative histories for speech, hearing, or language impairments.

\section{Speaking Task}

Three speaking contexts were recorded from each participant: word ("BAba"), sentence ("Buy Bobby a puppy"), and paragraph ("Grandfather passage"). Participants were instructed to repeat the word and sentence tasks 10 times each at a comfortable rate and loudness. We tested different speaking contexts to determine if chin movement errors varied as a function of speaking tasks. The utterances "BAba" and "Buy Bobby a puppy" were selected because they contain bilabials, which are expected to maximally influence the motion of the chin in comparison with other phonemes that require little or no lower lip movement (Cooker, 1973).

\section{Pellet Location and Movement Tracking}

Chin and mandibular movement were tracked at 40 $\mathrm{Hz}$ using the $X R M B$, which registers the motion of radiodense pellets using computer-guided positioning of a narrowly focused x-ray (see Westbury, 1991). By convention, pellet positions were resampled to a rate of 160 samples per second. As illustrated in Figure 1, we tracked the movement of six pellets, four of which were affixed to the chin (chin angle [ChA], chin center $[\mathrm{ChC}]$, chin gnathion [ChG], chin right [ChR]) and two of which were affixed to the mandible (mandibular incisor $[\mathrm{MI}]$, mandibular molar $[\mathrm{MM}])$. The incisor pellet was cemented to the buccal surface of the mandibular incisor (MI), and the mandibular molar pellet (MM) was cemented to buccal surface of the junction between the first and second mandibular molars. ChA was placed posterior along the inferior border of the mandible approximately one quarter of the distance from the gnathion to the gonion (the posterior-inferior point on the angle of the mandible). Pellet $\mathrm{ChC}$ was placed on the face, midline at the most anterior and inferior point on the chin. Pellet ChG was placed under the chin on the skin overlying the most inferior point of the mandibular symphysis. Pellet ChR was placed on the face at approximately the same height as pellet $\mathrm{ChC}$ but about $2.5 \mathrm{~mm}$ to the right. The multiple pellet placements were used to identify the chin regions that contain the least amount of skin tracking error relative to pellet MI.

\section{Data Conditioning and Kinematic Representation}

Prior to analysis, the translatory and rotary components of two head-mounted pellets were used to obtain head-

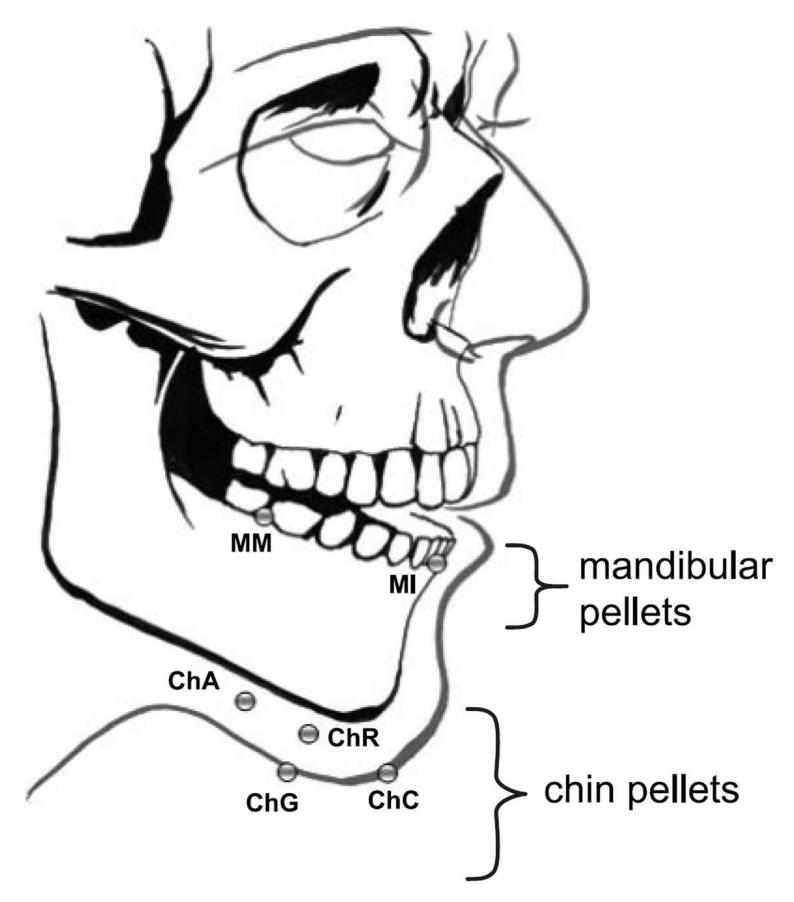

Note. As a way of determining the extent of skin-related error in the estimation of mandibular positions, comparisons were made between the movement of mandibular incisor (MI) and chin pellets. As a point of reference, the motion of mandibular molar (MM) was also compared with that of $\mathrm{MI} . \mathrm{ChA}=$ chin angle; $\mathrm{ChC}=$ chin center; $\mathrm{ChG}=$ chin gnathion; $\mathrm{ChR}=$ chin right.

Figure 1. Mandibular and chin pellet placements. 
referenced jaw positions. The positional data were subsequently expressed in a coordinate system that was defined by the maxillary occlusal plane (Westbury, 1994). The coordinate system origin was at the central maxillary incisors. The $y$-axis represented vertical movement and was normal to the maxillary occlusal plane; the $x$ axis represented anterior and posterior movement and was defined by the intersection of the midsagittal and maxillary occlusal planes. Prior to analysis, all signals were low-pass filtered $\left(\mathrm{f}_{\mathrm{lp}}=10 \mathrm{~Hz}\right)$ using a zero-phase forward and reverse digital filter.

The pellet motion paths for six repetitions of "BAba" produced by 1 adult participant are displayed in Figure 2. Each motion path is fitted with a 2-SD ellipse to emphasize the differing orientations among pellets in their primary axes of motion. Panel A in Figure 3 contains the mean-centered time histories of four pellets during "Buy Bobby a puppy" from 1 participant. The D signals represent the pellets' Euclidean distance from the maxillary-based origin, and the $X$ and $Y$ signals are positions relative to the $x$ - and $y$-axes of the maxillary occlusal plane, respectively. The time histories in Panel B of Figure 3 are amplitude-normalized versions of those displayed in Panel A. Visual inspection of the normalized traces provides a qualitative evaluation of the similarities and differences among pellet movement patterns regardless of differences in signal amplitude. Although these movement traces come from only 1 participant, the amplitude normalized data suggest that positional differences among mandibular and chin pellets cannot be accounted for only by linear scaling differences.

\section{Quantification of Chin Movement Error}

We performed two analyses to quantify the independence of chin and mandibular pellet motions. The first analysis quantified the absolute and relative spatial error between MI and the other pellets. If the chin is tightly coupled to the mandible during speech, the absolute error between MI and the chin pellets is expected to be small. For this analysis, the distance between MI and the other pellets was computed as a function of time across each speech record. For each pellet, distance signals were computed separately for the $X$ and $Y$ dimensions and for a signal (D) that represented the Euclidean distance from MI. The distance calculation effectively reexpressed the positional data from the maxillary-based coordinate system to a mandibular-based one with MI at the origin. The absolute error for each pellet was estimated by computing the standard deviation (rootmean-square of the distances) across each derived distance function (distance between each pellet and MI). Error estimates were intended to indicate the extent to which pellet motion varied from MI; however, because absolute error was statistically affected by a pellet's extent of movement and its distance from MI, relative error was calculated by dividing each signal's absolute error by its linear displacement.

Absolute and relative errors were also calculated for MM. Differences between the motions of MM and MI

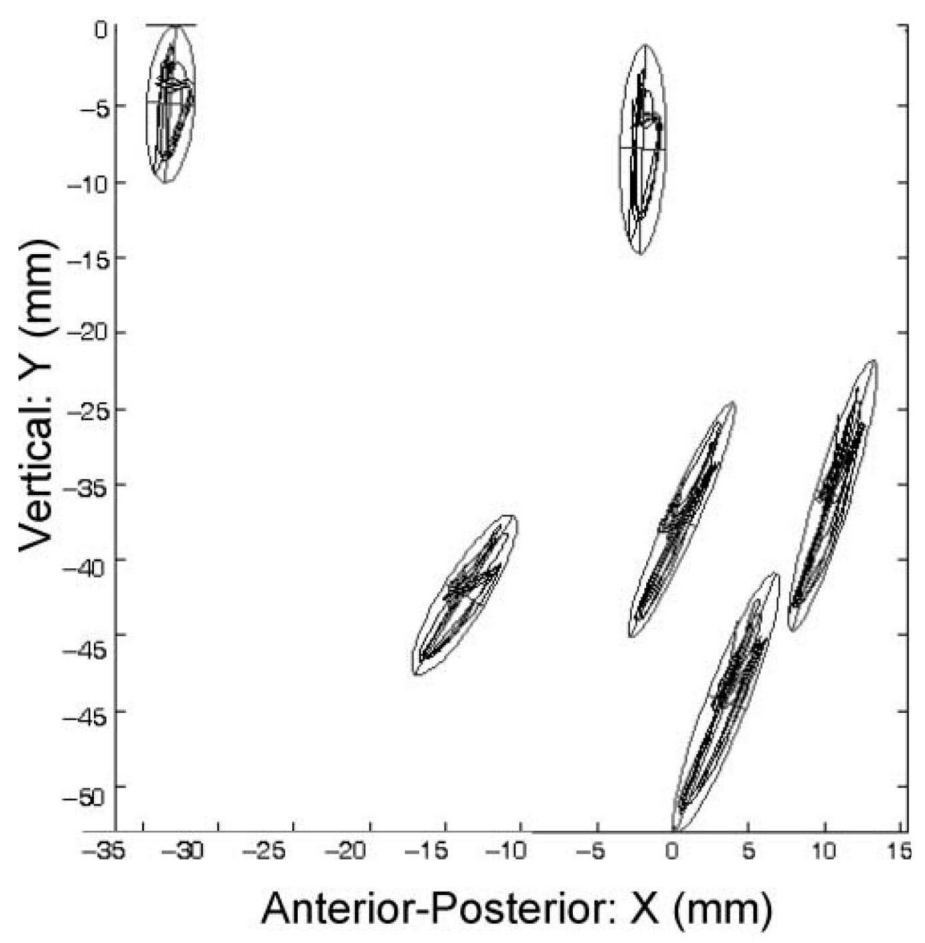

Figure 2. The motion paths of mandibular and chin pellets in the midsagittal plane. 
provided an estimate of the expected error between two pellets mounted on the mandible. Small differences between the movements of these pellets have several potential sources, including the limits in spatial resolution of XRMB tracking and the presence of planar distortions. Planar distortions arise from small translations and rotations of the head that are out of the midsagittal plane during data collection and from the projection of 3D motions and noncoplanar pellets onto the midsagittal plane.

We used a cross-correlation approach to quantify the independence of chin and mandibular pellet motions, regardless of differences in signal amplitude (see Green et al., 2000). It also provided a measure of asynchrony between MI and the other pellets (i.e., lag from MI). For each pellet, a time-series signal was extracted that represented its distance from the maxillary-based origin in the $X$ and $Y$ dimensions and as its Euclidean distance (D) from the maxillary origin (see Figure 3 ).

Peak coefficients (negative or positive) and their associated lags (the intervals between their movements) were derived from the cross-correlation functions com- puted among $\mathrm{X}, \mathrm{Y}$, and $\mathrm{D}$ signals of $\mathrm{MI}$ and the other pellets. In this analysis, weak pellet-movement coupling is inferred from low-peak cross-correlation coefficients and long lags (asynchronous movement); strong pellet movement coupling is inferred from high-peak crosscorrelation coefficients and short lags (near synchronous movement). Strong spatiotemporal coupling is expected for points located on a rigid object. Therefore, the observation of weak movement coupling among chin and MI pellets would suggest that chin points provide a poor representation of underlying mandibular movement.

\section{Statistical Treatment}

Mean relative error, cross-correlation coefficient, and lag values were subjected to a repeated two-way analysis of variance (ANOVA) having three levels of tasks (word, sentence, and paragraph) and five levels of pellets (MM, ChA, ChC, ChG, and ChR). Statistics were performed only on the relative error data and not on the absolute error data because, as stated previously, absolute error is expected to differ systematically across pellets due to
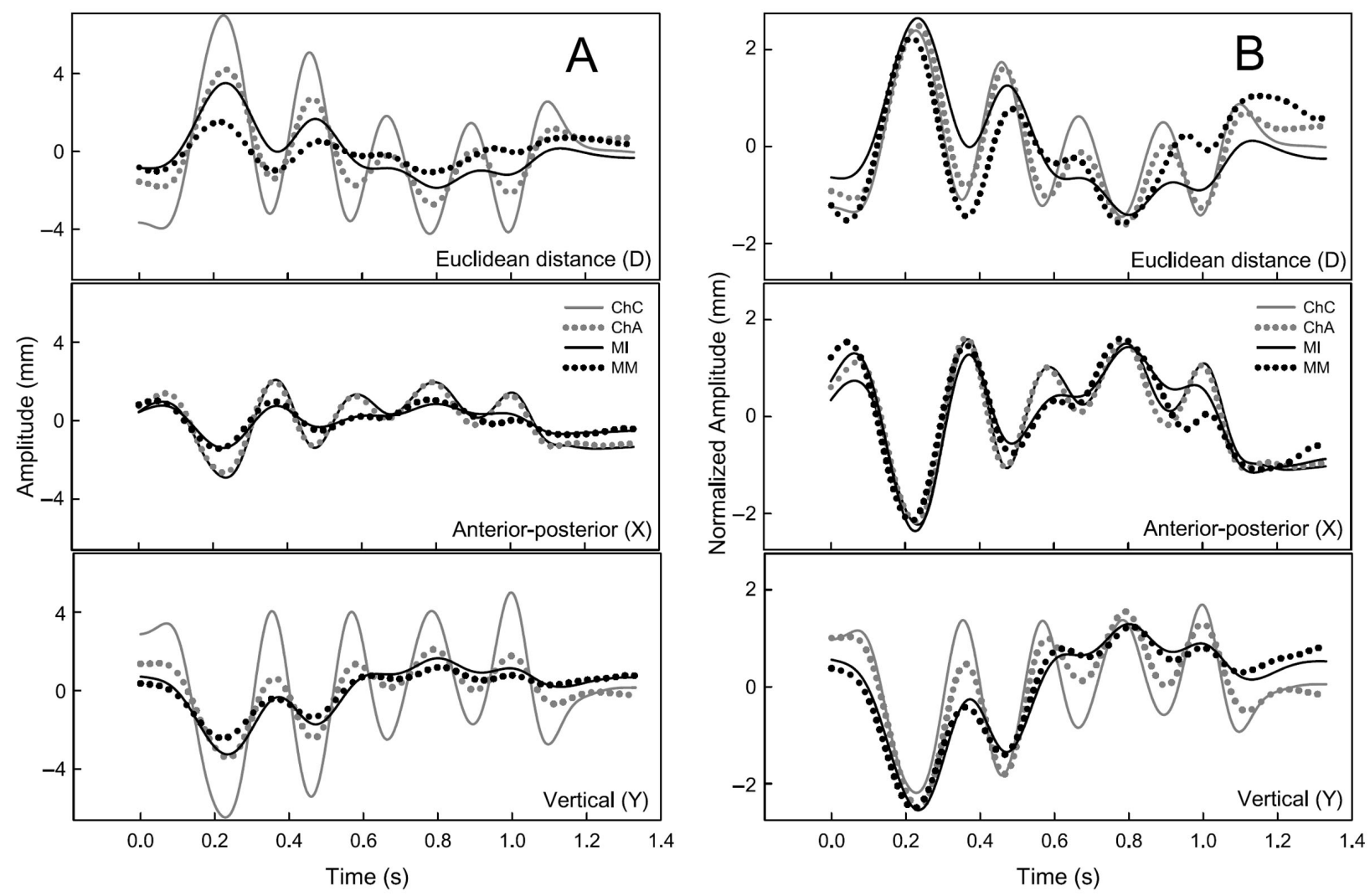

Note. Panel A contains the Euclidean distance (D), anterior-posterior (X), and vertical $(Y)$ time histories of four pellets during "Buy Bobby a puppy" from 1 participant. The time histories in Panel B are amplitude-normalized versions of those displayed in Panel A.

Figure 3. An example of mandibular and chin pellet motions during "Buy Bobby a puppy" from 1 participant. 
factors other than chin error. Prior to statistical analysis, relative error values, which were expressed as percentages, were transformed using the arcsine function; all absolute correlation values were converted into Fisher $z$ scores.

Differences among pellets in their relative errors, coefficients, and lags from MI were tested separately for each signal type (i.e., D, X, and $\mathrm{Y}$ ) using a one-way ANOVA. Statistical testing was performed on the participants' means computed across repetitions and tasks. Differences among the speaking tasks were tested using the same statistical model, but the data were averages computed across repetitions and pellets (excluding MM). Significant differences among participants were tested for each dependent variable using a repeated measures one-way ANOVA.

The Holm-Sidak and Student-Newman-Keuls methods were used for testing all pairwise multiple comparisons when significant differences were found. The Student-Newman-Keuls method was used for data that did not meet the assumption of normality. An alpha of $p<$ .05 was used for all statistical testing.

\section{Results}

\section{Positional Error}

The summary statistics for absolute and relative error across participants, pellets, and speaking tasks for signals $\mathrm{D}, \mathrm{X}$, and $\mathrm{Y}$ are shown in Tables 1, 2, and 3, respectively.

Signal effect. Error values of the D, X, and Y signals for each pellet are displayed in Tables 1, 2, and 3, respectively. Across tasks and pellets, relative error was significantly smaller for the D signal than it was for the $\mathrm{X}(q=$ $16.12, p<.05)$ and $Y(q=5.50, p<.05)$ signals. In general, the relative errors for the $X$ and $Y$ signals were at least twice as large as those for the D signal.

Pellet effect. The average absolute and relative errors for each pellet across participants and tasks are shown in Tables 1,2, and 3. The absolute error between the chin pellets and MI were approximately $1.27 \mathrm{~mm}$ or less for the D signal, $1.71 \mathrm{~mm}$ or less for the $\mathrm{X}$ signal, and 3.06 $\mathrm{mm}$ or less for the $\mathrm{Y}$ signals. The absolute error between MI and MM was small, approximately $0.19 \mathrm{~mm}$ for the $\mathrm{D}$ signal, $0.21 \mathrm{~mm}$ for the $\mathrm{X}$ signal, and $0.62 \mathrm{~mm}$ for the Y signal.

Post hoc comparisons revealed the following differences in relative error across pellets: (a) for the D signal, only pellet $\mathrm{ChC}$ exhibited significantly larger relative error than did MM, (b) for the $X$ signal, relative error was smaller for MM than for all chin pellets, and relative error was larger for pellet ChG than all of the other chin pellets, and (c) for the $\mathrm{Y}$ signal, relative error was smaller for MM than for all chin pellets, and pellet ChA exhibited significantly smaller relative error than all of the other chin pellets.

Task effect. The absolute and relative errors for the task variable, shown in Tables 1, 2, and 3, represent averages

Table 1. The mean and standard deviation of absolute error, relative error, and displacement for the Euclidian distance signal (D).

\begin{tabular}{|c|c|c|c|}
\hline Variable & $\begin{array}{l}\text { Absolute error (mm) } \\
\qquad M(S D)\end{array}$ & $\begin{array}{l}\text { Relative error }(\%) \\
\quad M(S D)\end{array}$ & $\begin{array}{c}\text { Displacement }(\mathrm{mm}) \\
M(S D)\end{array}$ \\
\hline Participant & $0.81(0.28)$ & $7.30(1.24)$ & $10.39(2.70)$ \\
\hline \multicolumn{4}{|l|}{ Pellet } \\
\hline MM vs. MI (1) & $0.19(0.10)$ & $6.09(2.54)$ & $3.52(1.79)$ \\
\hline ChA vs. MI (2) & $0.58(0.33)$ & $6.46(2.15)$ & $8.79(3.04)$ \\
\hline ChC vs. MI (3) & $1.27(0.53)$ & $8.71(2.76)$ & $14.10(4.23)$ \\
\hline ChG vs. MI (4) & $1.05(0.50)$ & $7.29(2.78)$ & 13.52 (4.37) \\
\hline ChR vs. MI (5) & $1.01(0.48)$ & $7.95(3.12)$ & $12.57(2.76)$ \\
\hline Post hoc & & $3>1$ & \\
\hline \multicolumn{4}{|l|}{ Task } \\
\hline Word (1) & $0.78(0.57)$ & $7.63(3.28)$ & $10.09(4.36)$ \\
\hline Sentence (2) & $0.88(0.61)$ & $8.06(2.86)$ & $10.69(4.44)$ \\
\hline Paragraph (3) & $0.76(0.38)$ & $6.21(1.83)$ & $11.63(3.81)$ \\
\hline Post hoc & & $2>3$ & \\
\hline
\end{tabular}

Displacement values are the linear distance (maximum-minimum position) across the entire file for each pellet that is being compared to MI. Average error estimates for the task and participant variables do not include the results of MM versus MI. The number in parentheses after each row beneath "Pellet" and "Task" refers to the number used for illustrating significant differences in the subsequent row titled "Post hoc." $\mathrm{MI}=$ mandibular incisor; $\mathrm{MM}=$ mandibular molar; $\mathrm{ChA}=$ chin angle; $\mathrm{ChC}=$ chin center; $\mathrm{ChG}=$ chin gnathion; $\mathrm{ChR}=$ chin right. 
Table 2. The mean and standard deviation of absolute error, relative error, and displacement for the anterior-posterior dimension signal $(\mathrm{X})$.

\begin{tabular}{llll}
\hline Variable & $\begin{array}{c}\text { Absolute error }(\mathrm{mm}) \\
M(S D)\end{array}$ & $\begin{array}{c}\text { Relative error }(\%) \\
M(S D)\end{array}$ & $\begin{array}{c}\text { Displacement (mm) } \\
M(S D)\end{array}$ \\
\hline $\begin{array}{l}\text { Participant } \\
\text { Pellet }\end{array}$ & $1.08(0.37)$ & $15.14(1.57)$ & $5.96(1.93)$ \\
$\quad$ & & \\
MM vs. MI (1) & $0.21(0.11)$ & $7.49(3.58)$ & $3.03(1.61)$ \\
ChA vs. MI (2) & $1.11(0.50)$ & $16.26(5.70)$ & $6.10(2.46)$ \\
ChC vs. MI (3) & $1.16(0.48)$ & $16.65(5.65)$ & $6.31(2.34)$ \\
ChG vs. MI (4) & $1.71(0.70)$ & $18.71(6.18)$ & $6.39(3.11)$ \\
ChR vs. MI (5) & $1.25(0.63)$ & $16.60(5.62)$ & $6.52(2.76)$ \\
Post hoc & & $1<$ all chin; $4>2,3,5$ & \\
Task & & & \\
$\quad$ Word (1) & $1.06(0.65)$ & $15.88(5.12)$ & $6.45(2.66)$ \\
$\quad$ Sentence (2) & $0.79(0.36)$ & $9.37(2.65)$ & $11.63(1.91)$ \\
$\quad$ Paragraph (3) & $0.76(0.38)$ & $6.21(1.83)$ & \\
Post hoc & & $3<1,2 ; 1>2$ & \\
\hline
\end{tabular}

Displacement values are the linear distance (maximum-minimum position) across the entire file for each pellet that is being compared to MI. Average error estimates for the task and participant variables do not include the results of MM versus MI. The number in parentheses after each row beneath "Pellet" and "Task" refers to the number used for illustrating significant differences in the subsequent row titled "Post hoc."

across participants and chin pellets. Post hoc comparisons revealed the following differences across speaking tasks: (a) for the D signal, relative error was significantly larger for the sentence task than for the paragraph task, (b) for the $X$ signal, relative error was significantly smaller for the paragraph task than for the word task and sentence tasks, and relative error was significantly larger for the word task in comparison with the sentence task, and (c) for the $\mathrm{Y}$ signal, relative error was significantly smaller for the paragraph task than for the word and sentence tasks.

Table 3. The mean and standard deviation of absolute error, relative error, and displacement for the vertical dimension signal $(\mathrm{Y})$.

\begin{tabular}{lllc}
\hline Variable & $\begin{array}{c}\text { Absolute error }(\mathrm{mm}) \\
M(S D)\end{array}$ & $\begin{array}{c}\text { Relative error }(\%) \\
M(S D)\end{array}$ & $\begin{array}{c}\text { Displacement }(\mathrm{mm}) \\
M(S D)\end{array}$ \\
\hline $\begin{array}{l}\text { Participant } \\
\text { Pellet }\end{array}$ & $1.89(0.45)$ & $15.32(1.50)$ & $10.72(2.53)$ \\
$\quad$ & & \\
MM vs. MI (1) & $0.62(0.26)$ & $10.58(3.23)$ & $5.78(2.52)$ \\
ChA vs. MI (2) & $1.00(0.61)$ & $13.06(5.81)$ & $15.16(2.46)$ \\
ChC vs. MI (3) & $3.06(1.03)$ & $18.51(5.81)$ & $13.39(4.00)$ \\
ChG vs. MI (4) & $2.60(0.92)$ & $17.37(5.59)$ & $12.24(3.36)$ \\
$\quad$ ChR vs. MI (5) & $2.27(0.75)$ & $17.09(5.08)$ & \\
Post hoc & & $1<$ all chin; $2<3,4,5$ & \\
Task & & & $10.49(4.72)$ \\
$\quad$ Word (1) & $1.99(1.28)$ & $17.97(6.04)$ & $10.80(4.74)$ \\
$\quad$ Sentence (2) & $1.96(1.21)$ & $10.90(2.25)$ & $11.97(4.23)$ \\
$\quad$ Paragraph (3) & $1.38(0.69)$ & $3<1,2$ & \\
Post hoc & &
\end{tabular}

Displacement values are the linear distance (maximum-minimum position) across the entire file for each pellet that is being compared to MI. Average error estimates for the task and participant variables do not include the results of MM versus MI. The number in parentheses after each row beneath "Pellet" and "Task" refers to the number used for illustrating significant differences in the subsequent row titled "Post hoc." 


\section{Spatiotemporal Pattern Error}

Coefficient values were obtained from the pairwise cross-correlation between MI and each pellet. These values provided an index of the degree of similarity between the normalized movement patterns of each pellet and that of MI.

Signal effect. Correlations for the $\mathrm{X}$ and $\mathrm{Y}$ signals (Chin Pellets $x$ MI) were significantly stronger than were those for the D signals $(q=12.1 ; 7, p<.05)$, and the $\mathrm{X}$ signals were also more strongly correlated with those of MI than were the $\mathrm{Y}$ signals $(q=11.2, p<.05)$.

Pellet effect. The means and standard deviations for the coefficient values of each pellet are shown in Table 4. Across signals, average coefficients ranged from 0.80 to 0.98 , suggesting a moderate to high degree of similarity between the movements of chin and mandibular pellets.

Post hoc comparisons revealed the following differences across pellets: For the D signal, the movement patterns of ChA were more strongly correlated with $\mathrm{MI}$ than were those of $\mathrm{MM}, \mathrm{ChC}$, or $\mathrm{ChR}$, and $\mathrm{ChG}$ was more strongly correlated with $\mathrm{MI}$ than was $\mathrm{ChC}$. For the $X$ signal, pellet MM was more strongly correlated with MI than were all of the chin pellets. For the Y signal, MM was more strongly correlated with MI than were any of the chin pellets. ChA was more strongly correlated with MI than were the other chin pellets. In general, correlation differences among the chin pellets were very small but, in some cases, still statistically significant.

Task effect. The means and standard deviations for the correlation values of each task are shown in Table 4. In general, correlation values decreased with an increase in utterance length (i.e., word > sentence $>$ paragraph). Post hoc comparisons revealed that for all the signals, coupling between chin and mandibular pellets was larger for the word task than for either the sentence or the paragraph tasks.

\section{Temporal Error}

The means and standard deviations for the absolute lag values between each pellet and MI are shown in Table 4. In general, the lag from MI was short for all chin pellets - less than $8 \mathrm{~ms}$. In addition, the standard deviation values within each participant were almost all larger than the mean values, suggesting a high degree of variability within and across participants.

Signal effect. Post hoc comparisons revealed that the $\mathrm{X}$ and $Y$ signals exhibited significantly shorter lags $(q=12.1$, $p<.05)$ from MI than did the D signal $(q=7.2, p<.05)$.

Pellet effect. Post hoc comparisons revealed several small but significant differences among pellets in their lag time from MI: For the D signal, MM exhibited significantly longer lags than did all of the chin pellets. For the $\mathrm{X}$ signal, $\mathrm{MM}$ exhibited a significantly longer lags than did the chin pellets, $\mathrm{ChC}$ and $\mathrm{ChR}$. For the $\mathrm{Y}$ signal, only pellet $\mathrm{ChC}$ exhibited significantly longer lags than did pellet ChA.

Task effect. There were no significant task effects for lag from MI.

Table 4. The mean and standard deviation of cross-correlation coefficients (r) and lags (ms) for the D, X, and $Y$ signals as a function of participant, pellet, and speaking task.

\begin{tabular}{|c|c|c|c|c|c|c|}
\hline \multirow[b]{2}{*}{ Variable } & \multicolumn{3}{|c|}{ Coefficient (r) } & \multicolumn{3}{|c|}{ Lag (ms) } \\
\hline & $\mathrm{D}$ & $x$ & $\mathrm{Y}$ & $\mathrm{D}$ & $\mathrm{X}$ & $\mathrm{Y}$ \\
\hline Participant & $0.84(0.05)$ & $0.93(0.03)$ & $0.87(0.05)$ & $12.61(7.06)$ & 4.33 (1.96) & $5.76(3.78)$ \\
\hline \multicolumn{7}{|l|}{ Pellet } \\
\hline MM (1) & $0.82(0.13)$ & $0.97(0.04)$ & $0.98(0.02)$ & 35.49 (50.93) & 7.45 (11.83) & $2.66(3.62)$ \\
\hline ChA (2) & $0.88(0.08)$ & $0.91(0.09)$ & $0.89(0.08)$ & 4.85 (14.64) & $3.93(5.26)$ & $3.93(5.30)$ \\
\hline ChC (3) & $0.81(0.12)$ & $0.93(0.06)$ & $0.80(0.11)$ & $7.99(17.20)$ & $2.70(4.60)$ & $7.65(11.84)$ \\
\hline ChG (4) & $0.86(0.10)$ & $0.90(0.08)$ & $0.84(0.10)$ & $5.70(11.18)$ & $4.61(12.05)$ & 6.05 (11.13) \\
\hline ChR (5) & $0.84(0.11)$ & $0.93(0.08)$ & $0.83(0.11)$ & 7.65 (15.42) & $2.97(4.84)$ & 7.48 (8.13) \\
\hline Post hoc & $2>1,3,5 ; 4>3$ & $1>$ all chin & $1>$ all chin; $2>3,4,5$ & $1>$ all chin & $1>3,5$ & $3>2$ \\
\hline \multicolumn{7}{|l|}{ Task } \\
\hline Word (1) & $0.90(0.08)$ & $0.96(0.04)$ & $0.92(0.08)$ & $12.62(26.66)$ & $4.52(8.89)$ & 6.05 (11.36) \\
\hline Sentence (2) & $0.80(0.12)$ & $0.90(0.09)$ & $0.83(0.12)$ & $13.85(33.90)$ & 3.95 (8.98) & $5.17(5.48)$ \\
\hline Paragraph (3) & $0.76(0.11)$ & $0.90(0.08)$ & $0.81(0.11)$ & $4.74(4.22)$ & $4.94(4.79)$ & $4.67(4.35)$ \\
\hline Post hoc & $1>2,3$ & $1>2,3$ & $1>2,3$ & NS & NS & NS \\
\hline
\end{tabular}

The number in parentheses after each row beneath "Pellet" and "Task" refers to the number used for illustrating significant differences in the subsequent row titled "Post hoc." NS = nonsignificant. ${ }^{*} p<.05$. 


\section{Discussion}

Most of the existing knowledge of jaw performance during speech and chewing has come from recordings of chin surface landmarks. This investigation examined the extent to which the motion of the chin accurately represents that of the underlying mandible. The accuracy of tracking varied depending on the pellets' location on the chin, the speaking task, and the representative signal. The present findings suggest that, on average, the position of chin pellets can be used to estimate those of the mandible within an absolute and relative error of approximately $0.81 \mathrm{~mm}$ and $7.3 \%$, respectively (see participants' mean for absolute error of D signal in Table 1). As anticipated, the D signal provided the most representative estimate of spatial error because the $X$ and $Y$ signals were significantly affected by projection distortions. The present findings would be expected to generalize to a larger population of young adults because acrossparticipant differences were relatively small for most of the error measures. Future work is required, however, to determine if chin-tracking error varies across individuals depending on their facial tissue elasticity, body fat composition, and movement extent. In particular, these factors may affect chin mobility in aged individuals and young children.

\section{Spatial and Temporal Error}

Of course, the acceptable amount of chin surface tracking error depends on the purpose of an investigation. The issue of chin-surface target error may be irrelevant for studies that are specifically interested in facial movement rather than mandibular movement. For studies of kinematic changes across different tasks or during development, the $7.3 \%(0.81 \mathrm{~mm})$ positional error observed for chin tracking is probably acceptable for detecting statistical differences. However, caution is recommended when using data from multiple chin pellets for obtaining jaw-corrected lower lip positions because chin surface errors may be additive. Additional work is needed to better understand this potential problem. The observed amount of error also may not be tolerable for modeling the mechanical aspects of jaw motion.

As in previous investigations, movements of the chin were larger than were those of the mandible during speech (Cooker, 1973; Kuehn et al., 1980). In contrast, prior studies on chewing have shown that chin movement underestimates the amplitude of mandibular movements. Jemt and Hedgård (1982) reported a difference between vertical displacements and velocity between $12 \%$ and $20 \%$. The disparity between the findings on chewing and speech probably relates to differences in lower lip function for each task. During chewing, the lower lip seals the anterior oral cavity to prevent leakage and, therefore, maintains a relatively stationary position. During speech, the lower lip assumes a wide variety of positions and shapes. The large excursions of the lower lip during speech may stretch and compress chin tissue. The tissues of the chin may also move in response to inertial forces of the underlying mandible.

Factors other than skin movement error may have contributed to the observed error in chin pellet positions in this investigation. Specifically, there was no attempt to correct the positional data for the effects of off midsagittal plane head movements, and differences among pellets in their relative distance between the x-ray pinhole and the image plane. Westbury (1991) provides an example showing $\pm 0.4 \mathrm{~mm}$ error for two pellets $(20 \mathrm{~mm}$ apart) moving $\pm 10 \mathrm{~mm}$ with respect to an image-plane pinhole distance of $500 \mathrm{~mm}$. The potential for these distortions is particularly relevant for the present study because distance from pinhole varied among pellets depending on their location on the chin and mandible. For example, pellets $\mathrm{ChR}$ and ChA were positioned lateral to MI. Pellet MM was also positioned lateral to MI and, therefore, the observed error between MM and MI provided a general estimate of the magnitude of the distortions due to head movements and pinhole-distance differences in the absence of skin movement artifacts. The findings presented in Table 1 suggest that error from this source was very small (i.e., $<0.2 \mathrm{~mm}$ ) and that there were no obvious pinhole-distance effects among pellets.

Pellet effects. The movements of MI and the chin pellets were nearly synchronous (i.e., lags shorter than 8 $\mathrm{ms}$ ). These findings suggest that measures of chin movement timing provide an acceptable representation of those of the mandible. It has also been reported that chin movements also preserve the timing of mandibular movements during cyclic chewing (Jemt \& Hedgård, 1982; Chmielewski, Feine, Maskawi, \& Lund, 1994; Häggman-Henrikson, Eriksson, Nordh, \& Zafar, 1998; Zafar, Nordh, \& Eriksson, 2002).

The correlation analyses indicate the degree to which the details of the shape of chin and mandibular movement traces are similar-regardless of differences in signal amplitude. Correlations varied depending on the representative signal, pellet location, and speaking task. Across pellets and signals, the correlation analysis yielded coefficients of 0.80 or greater, which suggests that chin movement traces were similar in shape but not identical to those of the underlying mandible. Among the chin pellets, the pellet ChA, which was located farthest from the most anterior and inferior point on the chin, was the most strongly correlated with the mandibular pellet. Thus, this region of the chin was the least affected by lower lip movement. Unfortunately, of all the pellets, ChA may be the most difficult to track because of its lateral location on the face. 
Under the best conditions, it would be expected that the movements of the incisor (MI) and molar (MM) pellets would be very similar because both points are attached to the rigid mandible. In the present study, the absolute difference between these pellets was very small $(<0.2 \mathrm{~mm})$, which is near the spatial resolution of the XRMB system (Westbury, 1994). When expressed as relative error, however, the difference between these two pellets was as large as $6 \%$ for the D signal and $10.58 \%$ for the $\mathrm{Y}$ signal.

Signal effects. Differences among studies in the degree of chin movement error depend not only on whether speech or chewing is being investigated but also on how the movement signals are being represented. The present findings revealed the strengths and weaknesses of each representation. Specifically, the D signal contained $50 \%$ less relative error than the $X$ and $Y$ signals; however, correlations with MI were significantly weaker for the $\mathrm{D}$ than for the $\mathrm{X}$ and $\mathrm{Y}$ signals.

Projection distortion may have inflated relative error estimates for the $X$ and $Y$ signals. Specifically, visual inspection of the motion paths for each subject revealed that the primary axis of motion of chin pellets was typically rotated clockwise relative to those of the mandibular pellets (see, e.g., Figure 2). In the head-based coordinate system used in this study, this anterior rotation will have the effect of increasing $X$ displacements and decreasing $Y$ displacements of the chin pellets relative to the more vertically oriented mandibular pellets.

The high correlations between MI and the chin pellets suggested, however, that the differences in motion path orientation between mandibular and chin pellets did not significantly alter the shape of the $X$ and Y movement patterns. Shape may have been preserved in the unidimensional representation because, as illustrated in Figure 2, mandibular and chin movements during speech intersected both the $\mathrm{X}$ and $\mathrm{Y}$ dimensions of the head-based coordinate system. In contrast, the Euclidean distance transform (see Figure 3, Panel A) produced small positional deviances that apparently distorted the shape of the movement pattern, which created some surprising results for the crosscorrelation analysis. For example, for the D signal, the movement patterns of ChA were more strongly correlated with MI than were those of $\mathrm{MM}, \mathrm{ChC}$, or $\mathrm{ChR}$. Moreover, the temporal lags between MI and MM were nearly 5 times larger than those of the chin pellets, and the correlations among these pellets were also lower than those of the chin pellets.

In summary, these findings suggest the Euclidean distance transform minimized spatial error but had a greater effect on the shape of movement patterns. Conversely, the unidimensional representations of chin movement (i.e., $X$ and $Y$ ) contained more spatial error but better preserved the shape of movement patterns than did the D signal. One practical interpretation of this finding is that the D signal is the most appropriate when taking measures that depend on spatial accuracy relative to the mandible, whereas the $X$ and $Y$ signals may yield more accurate results for correlation-based analyses.

Task effect. The task data suggest that the coupling between MI and the chin pellets is strongest for short utterances containing low vowels. Relative error was, however, smaller for the paragraph task than for the sentence task. The data in Table 1 suggest that this task difference is primarily due to differences in displacement rather than absolute error. The sentence task may have contained the greatest amount of error because it contained relatively more bilabial sounds than did the paragraph task.

\section{Conclusion}

The acceptable amount of chin surface tracking error will depend on the specific purpose of a particular study. If the objective of an investigation is to determine performance differences across different populations and speaking conditions, the issue of chin surface tracking error may be entirely irrelevant. The $7.3 \%(0.8 \mathrm{~mm})$ positional error observed for chin tracking in this study is considered acceptable for descriptive studies of oromotor behavior, particularly in situations where mandibular placements are not practical (e.g., young children or edentulous adults). The observed amount of error, however, may not be tolerable for fine-grained analyses of mandibular biomechanics. In these cases, investigators may choose to use alternative methods to track the jaw or track a marker located outside the mouth that is affixed to a dental splint (e.g., Gracco \& Löfqvist, 1994; Perkell \& Zandipour, 2002). Moreover, because of potential differences in tissue elasticity as a function of age, the extent to which these findings generalize to infants and aged individuals remains in question. Finally, caution is recommended when data from multiple chin pellets are used for obtaining jaw-corrected lower lip positions because chintracking errors may be additive.

The present findings suggest that the following procedures can be implemented to maximize the extent to which chin motions represent those of the underlying mandible: (a) the chin marker should be positioned off the fleshy region of the chin that is directly below the lower lip, (b) the Euclidean distance signal should be used when performing spatial analyses, and (c) a unidimensional signal should be used when performing a correlation-based analysis. 


\section{Acknowledgments}

This research was supported by Grants R03DC04643 and R01 DC06463 (awarded to the first author) and R01 DC000822 (awarded to the fourth author) from the National Institute on Deafness and Other Communication Disorders and by the Barkley Trust. We would like to acknowledge Pascal van Lieshout, Antje Mefferd, Ignatius Nip, Gary Weismer, and Yana Yunusova for providing feedback on early drafts of this article; David Wilson for computer programming; Mike Jackson for preparing the artwork; Carl Johnson for data collection and postprocessing; and John Westbury for providing access to the XRMB facility.

\section{References}

Abbs, J., \& Gilbert, B. (1973). A strain gauge transduction system for lip and jaw motions in two dimensions: Design criteria and calibration data. Journal of Speech and Hearing Research, 16, 248-256.

Abbs, J. H., Nadler, R. D., \& Fujimura, O. (1988). X-ray microbeams track the shape of speech. SOMA, 2, 29-34.

Ahlgren, J. (1966). Mechanism of mastication: A quantitative cinematographic and electromyographic study of mastication movements in children, with special reference to occlusion of the teeth. Acta Odontologica Scandinavica, 24, 5-109.

Barlow, S. M., Cole, K. J., \& Abbs, J. H. (1983). A new head-mounted lip-jaw movement transduction system for the study of motor speech disorders. Journal of Speech and Hearing Research, 26, 283-288.

Caruso, A. J., Stanhope, S. J., \& McGuire, D. A. (1989). New technique for acquiring three-dimensional orofacial nonspeech movements. Dysphagia, 4, 127-132.

Chmielewski, W., Feine, J. S., Maskawi, K., \& Lund, J. P. (1994). Recording mandibular movements using chin markers (abstract). Journal of Dental Research, 73, 114.

Cooker, H. S. (1973). On the problem of tracking mandibular movements. Speech Transmission Laboratory Quarterly Progress and Status Report, 2-3, 1-12.

DeNil, L. F., \& Abbs, J. H. (1991). Influence of speaking rate on the upper lip, lower lip, and jaw peak velocity sequencing during bilabial closing movements. The Journal of the Acoustical Society of America, 89, 845-849.

Edwards, J., \& Harris, K. S. (1990). Rotation and translation of the jaw during speech. Journal of Speech and Hearing Research, 33, 550-562.

Gracco, V. L. (1988). Timing factors in the coordination of speech movements. Journal of Neuroscience, 8, 4628-4639.
Gracco, V. L. (1994). Some organizational characteristics of speech movement control. Journal of Speech and Hearing Research, 37, 4-27.

Gracco, V. L., \& Löfqvist, A. (1994). Speech motor coordination and control: Evidence from lip, jaw, and laryngeal movements. The Journal of Neuroscience, 14, 6585-6597.

Green, J. R., Moore, C. A., Higashikawa, M., \& Steeve, R. W. (2000). The physiologic development of speech motor control: Lip and jaw coordination. Journal of Speech, Language, and Hearing Research, 43, 239-255.

Green, J. R., Moore, C. A., \& Reilly, K. J. (2002). The sequential development of jaw and lip control for speech. Journal of Speech, Language, and Hearing Research, 45, 66-79.

Green, J. R., \& Wilson, E. M. (2006). Spontaneous facial motility in infancy: A 3D kinematic analysis. Developmental Psychobiology, 48, 16-28.

Häggman-Henrikson, B., Eriksson, P. O., Nordh, E., \& Zafar, H. (1998). Evaluation of skin- versus teeth-attached markers in wireless optoelectronic recordings of chewing movements in man. Journal of Oral Rehabilitation, 25, 527-534.

Hamlet, S. L., \& Stone, M. (1978). Compensatory alveolar consonant production produced by wearing a dental prosthesis. Journal of Phonetics, 6, 227-248.

Hixon, T. J. (1971). An electromagnetic method for transducing jaw movements during speech. The Journal of the Acoustical Society of America, 49, 603-606.

Holden, J. P., Stanhope, S. J., \& Orsini, J. A. (1994). Skeletal motion estimates: Effect of surface target techniques. Proceedings of the Second World Congress of Biomechanics Amsterdam, 2, 372.

Jemt, T., \& Hedgård, B. (1982). The relative movements of the chin and the mandible during chewing. Journal of Oral Rehabilitation, 9, 253.

Kelso, J. A. S., Vatikiotis-Bateson, E., Saltzman, E. L., \& Kay, B. (1985). A qualitative dynamic analysis of reiterant speech production: Phase portraits, kinematics, and dynamic modeling. The Journal of Acoustical Society of America, 77, 266-280.

Kuehn, D. P., Reich, A. R., \& Jordan, J. E. (1980). A cineradiographic study of chin marker positioning: Implications for the strain gauge transduction of jaw movement. The Journal of the Acoustical Society of America, 67, 1825-1827.

McFarland, D. H., \& Lund, J. P. (1995). Modification of mastication and respiration during swallowing in the adult human. Journal of Neurophysiology, 74, 1509-1517.

Müller, E. M., \& Abbs, J. H. (1979). Strain gauge transduction of lip and jaw motion in the midsagittal plane: Re- 
finement of a prototype system. The Journal of the Acoustical Society of America, 65, 481-486.

Ostry, D. J., \& Munhall, K. G. (1994). Control of jaw orientation and position in mastication and speech. Journal of Neurophysiology, 71, 1528-1545.

Perkell, J., Cohen, M., Svirsky, M., Matthies, M., Garabieta, I., \& Jackson, M. (1992). Electro-magnetic midsagittal articulometer (EMMA) systems for transducing speech articulatory movements. The Journal of the Acoustical Society of America, 92, 3078-3096.

Perkell, J. S., \& Zandipour, M. (2002). Economy of effort in different speaking conditions. II. Kinematic performance spaces for cyclical and speech movements. The Journal of the Acoustical Society of America, 112, 1642-1651.

Shaiman, S. (2002). Articulatory control of vowel length for contiguous jaw cycles: The effects of speaking rate and phonetic context. Journal of Speech, Language, and Hearing Research, 45, 663-675.

Sharkey, S. G., \& Folkins, J. W. (1985). Variability of lip and jaw movements in children and adults: Implications for the development of speech motor control. Journal of Speech and Hearing Research, 28, 8-15.

Smith, A., Goffman, L., Zelaznik, H. N., Ying, G., \& McGillem, C. (1995). Spatiotemporal stability and patterning of speech movement sequences. Experimental Brain Research, 104, 493-501.

Smith, B. L., \& McLean-Muse, A. (1987). An investigation of motor equivalence in children. The Journal of the Acoustical Society of America, 82, 837-842.
Stone, M. (1981). Evidence for a rhythm pattern in speech production: Observations of jaw movement. Journal of Phonetics, 9, 109-120.

Sussman, H. M., MacNeilage, P. F., \& Hanson, R. J. (1973). Labial and mandibular dynamics during the production of bilabial consonants: Preliminary observations. Journal of Speech and Hearing Research, 16, 397-420.

Sussman, H. M., \& Smith, K. U. (1970). Transducer for measuring mandibular movements. The Journal of the Acoustical Society of America, 48, 857-858.

Throckmorton, G. S., Buschang, B. H., Hayasaki, H., \& Phelan, T. (2001). The effects of chewing rates on mandibular kinematics. Journal of Oral Rehabilitation, 28, 328-334.

Vatikiotis-Bateson, E., \& Ostry, D. J. (1995). An analysis of the dimensionality of jaw motion in speech. Journal of Phonetics, 23, 101-117.

Westbury, J. R. (1991). The significance and measurement of head position during speech production experiments using the X-ray microbeam system. The Journal of the Acoustical Society of America, 89, 1782-1791.

Westbury, J. R. (1994). X-ray microbeam speech production database user's handbook. Madison, WI: University of Wisconsin Press.

Zafar, H., Nordh, E., \& Eriksson, P. O. (2002). Spatiotemporal consistency of human mandibular and head-neck movement trajectories during jaw opening-closing tasks. Experimental Brain Research, 146, 70-76. 